\title{
Taming the BFKL Intercept via Gluon Saturation
}

\author{
Alexander Kovner ${ }^{\mathrm{a}}$ and Urs Achim Wiedemann* b \\ ${ }^{a}$ Department of Mathematics and Statistics,University of Plymouth, \\ 2 Kirkby Place, Plymouth, PL4 8AA, U.K. \\ b Theory Division, CERN, CH-1211 Geneva 23, Switzerland
}

We show that the inclusion of parton density effects in the perturbative small-x evolution reduces the strength of the powerlike growth of total hadronic cross sections.

\section{Introduction}

At center of mass energies above $\sqrt{s} \approx 100 \mathrm{GeV}$, total hadronic cross sections $\sigma$ show a continuous rise which persists up to the highest energies explored so far and which is consistent with a gentle powerlike growth,

$\sigma \propto s^{\alpha_{P}}, \quad$ where $\quad \alpha_{P}=0.08$

However, at asymptotically high $\sqrt{s}$, hadronic cross sections satisfy the unitarity or Froissart bound, $\sigma \leq \frac{\pi}{m^{2}} \ln ^{2} \frac{s}{m^{2}}$ where $m$ is the smallest mass in the theory. Thus, the power law growth (11), parametrized by the soft pomeron intercept $\alpha_{P}$, can be valid only in a preasymptotic, though possibly large, kinematical regime. So far, this growth is not understood in the context of QCD. Indeed, perturbation theory in the LO BFKL framework does result in a powerlike growth

$\sigma \propto s^{\omega}, \quad$ where $\quad \omega=\frac{\alpha_{s}}{\pi} N_{c} 4 \ln 2 \gg \alpha_{P}$,

but the BFKL intercept $\omega$ is much larger than the experimentally observed one. Inspecting at large rapidity $t=\ln \frac{s}{m^{2}}$ the analytically known dependence of the BFKL density $\Phi\left(b, k, t, k_{0}\right)$ of gluons of momentum $k$ at impact parameter $b$ originating from a gluon of momentum $k_{0}$ at initial rapidity $t_{0}$, two different perturbative (non-unitary) growth mechanisms can be identified in the BFKL calculation:

1. Growth in density: For $b<b_{\text {diff }} \simeq e^{\sqrt{\alpha_{s}} t}$, one finds $\Phi(b) \sim e^{\omega t}$. For BFKL, this is the dominant growth mechanism, resulting in (2).

2. Growth in impact parameter space: For $b_{\text {spread }}=e^{\epsilon \alpha_{s} t}$, one finds $\Phi\left(b_{\text {spread }}\right) \sim e^{\left(\omega-\epsilon^{2} \alpha_{s}\right) t}$. For BFKL, this is a subleading growth mechanism. 
Here, we review arguments that including "gluon saturation", i.e. parton density effects, in the LO perturbative QCD calculation allows to regulate the dominant growth in density, thus taming in comparison to BFKL the non-unitary powerlike growth to $\sigma \propto s^{\epsilon \omega}$. This provides a perspective for a perturbative calculation of the soft pomeron intercept.

\section{The dipole-hadron total cross section}

For an explicit discussion of gluon saturation effects, we take recourse to a toy model of a hadronic collision. At initial rapidity $t_{0}$, we consider a perturbative $q \bar{q}$-dipole with quarks at transverse positions $x$ and $y$ colliding with a hadronic target. The dipole scattering probability is $N(x, y)$ [we also write $N\left(b=\frac{x+y}{2}, r=\frac{x-y}{2}\right)$ ], and the total cross section is obtained by integrating over the impact parameter, $\sigma\left(t_{0}\right)=2 \int d^{2} b N(b, r)$. To calculate the rapidity dependence of the cross section, one may work in the target rest frame where $t>t_{0}$ corresponds to boosting the projectile. This boost generates additional gluons which in the large $N_{c}$-limit translate into additional dipoles at different transverse positions. Pictorially:

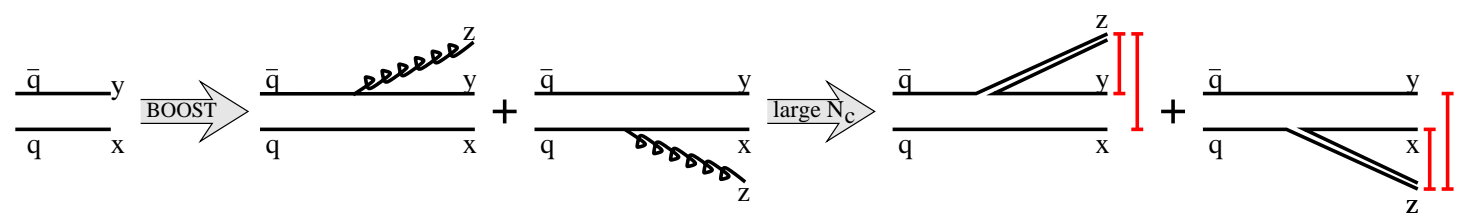

Calculating the interaction of this boosted dipole wave function with the hadronic target and taking the derivative with respect to the phase space opened up by the boost, one regains the Balitsky-Kovchegov equation [1]:

$\frac{d}{d t} N(x, y)=\bar{\alpha}_{s} \int \frac{d^{2} z}{2 \pi} \frac{(x-y)^{2}}{(x-z)^{2}(y-z)^{2}}[N(x, z)+N(y, z)-N(x, y)-N(x, z) N(z, y)]$.

This non-linear evolution equation differs from the linear BFKL equation by the addition of the last term $N(x, z) N(z, y)$ only. The physics contained in this modification of the BFKL equation is that of a double counting correction. Namely, for a projectile consisting of two dipoles $(x, z)$ and $(y, z)$, the scattering probability is the sum of the individual scattering probabilities minus the probability that both dipoles scatter:

$N(\{x, z\} ;\{y, z\})=N(x, z)+N(y, z)-N(x, z) N(z, y)$.

The remaining fourth term $N(x, y)$ in (3) is a virtual correction required by the proper normalization of the projectile wavefunction $|q \bar{q}\rangle+|q \bar{q} g\rangle$. In conclusion, the BK evolution equation (3) can be viewed in the target rest frame as BFKL evolution of the boosted projectile but supplemented by the double counting correction for the interaction of the evolved projectile wavefunction with the target. The swelling of the BK dipole projectile wavefunction in impact parameter space is thus known from BFKL. The density of dipoles of size $r$ at $b$ obtained by boosting the initial dipole of size $r_{0}$ to rapidity $t$ takes the form

$n\left(r, b, r_{0}, t\right)=\frac{32}{r^{2}} \frac{\ln \frac{16 b^{2}}{r_{0} r}}{\left(\pi a^{2} t\right)^{3 / 2}} \exp \left[\omega t-\ln \frac{16 r^{2}}{r_{0} r}-\frac{\ln ^{2} \frac{16 b^{2}}{r_{0} r}}{a^{2} t}\right]$ 
To determine the growth of the total cross section from this density, we consider the smallest dipole for which the scattering probability $N(r, b)$ is of order one for $b<R_{0}$ :
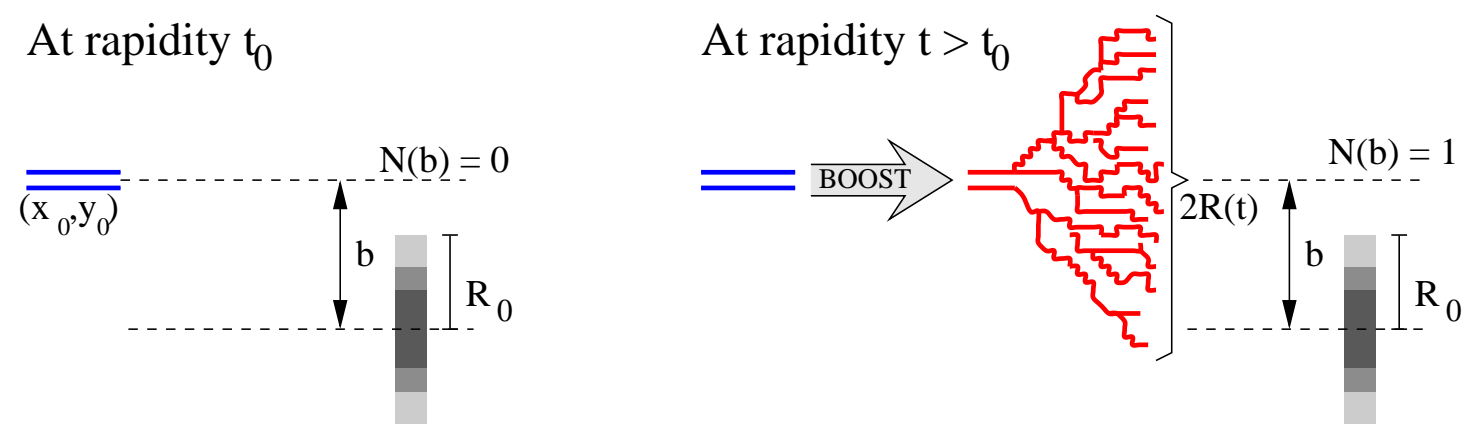

If after boosting the density of dipoles at $b$ becomes unity, then the scattering probability is of order unity as well. This establishes the non-unitary growth [2,3]

$\sigma(t)=\pi R^{2}\left(t, Q^{2}=1 / r^{2}\right) \quad$ with $\quad R^{2}\left(t, Q^{2}\right)=\frac{r_{0} r}{16} \exp [\epsilon t]$.

From (5), one finds for the BK intercept $\epsilon=\frac{\alpha_{s} N_{c}}{\pi} 7 \zeta(3)[-1+\sqrt{1+8 \ln 2 / 7 \zeta(3)}]$. This expression is parametrically correct but numerically questionable since (5) is based on a saddle point approximation valid for sufficiently small impact parameter only. Still, the numerical value $\epsilon=0.87 \omega$ is compatible with a numerical result of G. Salam [4] who finds a non-unitary growth of total cross sections with $\epsilon \approx 0.75 \omega$ in a simulation which encodes essentially the same physics as (31).

\section{Saturation without Unitarization: alternative derivations}

The above derivation was given in the target rest frame where the evolution resides in the projectile wavefunction. The same result can be obtained [2, [3] within Weigert's formulation where the evolution is ascribed to the target. This latter argument can be viewed as establishing the non-unitary growth of the hadronic cross section for a coloured scattering probe. Moreover, it was shown [0] that the total cross section violates unitarity for a colourless projectile as soon as it does so for a coloured one. These three arguments are independent of each other but consistent with each other and with Ref. [4]. They establish that while the BK equation ensures saturation of the scattering probability locally in impact parameter space (i.e. $N(b, r)<1$ for all $b$ ), it violates unitarization of the total cross section [2].

\section{Beyond the BK equation (BBK)}

In the target rest frame, the only density effect included in the BK equation (3) is the nonlinear double counting correction in (ब1). This correction sets in as soon as the number of gluons in the projectile state becomes large, even if the gluonic density may still be small. In addition, as soon as the partonic density in the projectile reaches a critical value of order $1 / \alpha_{s}$, one expects wavefunction saturation effects which further tame the 
growth of density in the projectile and are not included in (3). The rapidity scales for these different effects are:[2]

$t_{\mathrm{BFKL}} \propto \frac{1}{\alpha_{s} N_{c}}, \quad t_{\mathrm{BK}} \propto \frac{1}{\alpha_{s} N_{c} \ln \left(R_{0} / r_{0}\right)}, \quad t_{\mathrm{BBK}} \propto \frac{1}{\alpha_{s} N_{c} \ln \left(1 / \alpha_{s}\right)}$.

At rapidity $t_{\mathrm{BFKL}}$, the BFKL equation violates unitarity due to the growth in density. From (6) one finds that at rapidity $t_{\mathrm{BK}}$, the BK equation violates unitarity for a (small) dipole of initial size $r_{0}$ incident on a sufficiently extended target of size $R_{0}$. This violation is due to the growth of the projectile in transverse size. The applicability of the BK evolution, however, crucially depends on the nature of the target. If the target is thick enough, so that the scattering probability is parametrically larger than $\alpha_{s}$ [for a large nucleus of atomic number $A$, it is $O\left(A^{1 / 3} \alpha_{s}\right)$ ], and if the target is wide enough, so that saturation occurs before the projectile radius swells beyond that of the target, then there is an intermediate regime $t<t_{\mathrm{BK}}$ in which BK applies. However, if the target is a nucleon, neither one of these conditions is satisfied.

Wavefunction saturation effects should set in when the interaction probability of a dipole of size $r$ with another dipole of similar size is of order unity, $\alpha_{s} r^{2} n(r) \sim 1$. Taking the density growth exponential in rapidity, $r^{2} n(r)=e^{\omega t}$, this indicates that saturation effects beyond those included in the BK equation become relevant for $t>t_{\mathrm{BBK}}$.

It is reasonable to expect that projectile wavefunction saturation effects further diminish the intercept parameter of total cross sections beyond the exponent of the BK equation. The reason is that a tamed growth of the density in the projectile due to dipole interactions will result in a tamed growth in impact parameter. This suggests the hierarchy

$\omega_{\mathrm{BFKL}}>\omega_{\mathrm{BK}}>\omega_{\mathrm{BBK}}$

Thus, one is led naturally to conjecture that a non-linear generalization of the BFKL equation which goes beyond BK by taking into account wave function saturation effects ("Pomeron loops") provides a perturbative framework for the calculation of the soft Pomeron intercept, $\omega_{\mathrm{BBK}}=\alpha_{P}$. However, this requires that the perturbative growth mechanism occurs in the hadron on a scale smaller than the confinement scale at which the Froissart bound can be expected to arise as a consequence of non-perturbative physics. As explained in Ref. [3], a picture of the proton as consisting of three small black discs associated with the valence quarks is consistent with what is known about hadronic cross sections and allows for this possibility.

\section{REFERENCES}

1. I. Balitsky Nucl. Phys. B463 (1996) 99;

Yu. Kovchegov Phys. Rev. D60 (1999) 034008.

2. A. Kovner and U. A. Wiedemann, arXiv:hep-ph/0112140, Phys. Rev. D in press.

3. A. Kovner and U. A. Wiedemann, arXiv:hep-ph/0204277, Phys. Rev. D in press.

4. G.P. Salam, Nucl.Phys.B461 (1996) 512-538.

5. A. Kovner and U. A. Wiedemann, arXiv:hep-ph/0207335. 